\title{
Experiences in Data-Parallel Programming
}

\author{
TERRY W. CLARK, ${ }^{1, *}$ REINHARD v. HANXLEDEN, ${ }^{2,} \uparrow$ AND KEN KENNEDY ${ }^{2}$ \\ 'Texas Center for Advanced Molecular Computation, University of Houston, Houston, TX 77204, USA \\ ${ }^{2}$ Center for Research on Parallel Computation, Rice University, Houston, TX 77251, USA; e-mail: ken@rice.edu
}

\begin{abstract}
To efficiently parallelize a scientific application with a data-parallel compiler requires certain structural properties in the source program, and conversely, the absence of others. A recent parallelization effort of ours reinforced this observation and motivated this correspondence. Specifically, we have transformed a Fortran 77 version of Gromos, a popular dusty-deck program for molecular dynamics, into Fortran D, a data-parallel dialect of Fortran. During this transformation we have encountered a number of difficulties that probably are neither limited to this particular application nor do they seem likely to be addressed by improved compiler technology in the near future. Our experience with Gromos suggests a number of points to keep in mind when developing software that may at some time in its life cycle be parallelized with a data-parallel compiler. This note presents some guidelines for engineering data-parallel applications that are compatible with Fortran D or High Performance Fortran compilers. (c) 1997 John Wiley \& Sons, Inc.
\end{abstract}

\section{INTRODUCTION}

One concern often not foremost in a scientific programmer's mind at the outset of software development is parallelization. Yet, even for scientific applications developed for sequential execution, it is not unlikely that someone at sometime will parallelize the software. As it turns out, some programming styles are easier to parallelize than others. Moreover, for programs to yicld to the data-parallel approach of compilers for Fortran D [1] or High Performance Fortran (HPF) [2].

Received Finbruary 1995

Revised Jamuary 1996

*Present address: Pacific Nortliwest National Laboratorv, Richland. WA 90352. USA; -mail: twclark@emsl.pul.gov

fPresent address: Daimler-Benz AC. Responsive Systems. Berlin. Cermany: e-mail: vhanx@DBresearch-berlin.de

(c) 1997 by John Wiley \& Sons, Inc.

Scientific Programming. Vol. 6, pp. 153-158 (1997)

(C.C: $1058-9244 / 97 / 010153-06$ certain structural properties must be present in the software. Elements of a program style congruous with an HPF compiler include, for example, consistent distribution and use of data arrays and structured flow of control. It appears that writing such programs from the outset largely embraces good software engineering techniques (such as the structured-programming approach advocated by Dijkstra [3]). In this article, we discuss some of these requirements and provide guidelines for engineering data-parallel applications to be compatible with compilers for data-parallel languages. Alternatively, the observations presented here could also serve as guidelines for making an existing program suitable for data-parallel compilation. In the following, we will first outline some general guidelines. then illustrate them with short code examples, and finally give some additional suggestions to facilitate effective data parallelism.

One underlying idea of data-parallel languages. such as Fortran D or HPF, is that the user does not explicitly specify the parallelism inherent in a program, but instead annotates the program with directives on how to distribute the data, and lets the com- 
piler work from there. Performance and investmentpreserving independence from environment-specific details are two key objectives. The art of data-parallel programming might be defined as achieving the former without compromising the latter. For this correspondence, this also means that while the programmers should understand certain characteristics of data parallelism, they should not have to develop a style which will have an adverse effect on code readability, maintainability, or efficiency in nondata-parallel environments.

Performance depends on the degree of parallelism and on overheads, such as synchronization and communication costs. * Compilers for distributed memory architectures typically try to achieve parallelism by distributing loop iterations across processors. The first guideline for designing data-parallel programs should therefore be:

The flow of control should be structured; e.g., DOloops are preferable to GOTOs.

Distributing loop iterations is commonly driven by some heuristic for minimizing communication. such as the owner-computes rule [4] or variations thereof. This heuristic may fail even for "embarrassingly parallel" algorithms if the program expressing the algorithm obscures the parallelism by some (perhaps apparently unrelated) means. Even though there are many other issues erucial for achieving successful parallelization, realizing these points and designing program and data structures accordingly should in themselves go a long way toward good performance for many applications.

The perhaps most important guideline that should drive design decisions is:

\section{Loops and arrays should match.}

That is, in computationally intensive code regions. array subscripts and loop indices should be related to each other in a simple manner, allowing the compiler to derive a loop parallelization directly from an array distribution. To justify this guideline. let us briefly digress into the workings of a data-parallel compiler for distributed memory machines, such as the Fortran D compiler prototype at Rice University.

Assume the compiler is given a simple code segment

\footnotetext{
* Dynamic data distribution (redistribution at run-time) can result in considerable overhead. In this article. however. we address only those data distributions that may be interpreted statically
}

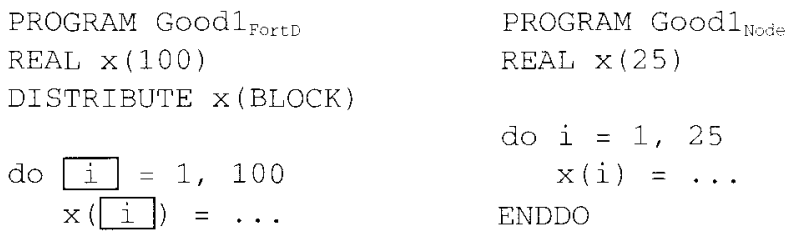

ENDDO

FIGURE 1 Simple example loop with matching array access; the Fortran D program (left) can be compiled into a node program (right) with reduced loop bounds (assuming $\left.N_{\mu m}=4\right)$. Distributed array and loop indices are framed.

as shown in Figure 1 on the left. Good1 $1_{\text {Fortd }}$. The Fortran D compiler will generate a node program Good1 $1_{\text {Nods. }}$ shown in Figure 1 on the right. which in turn will be compiled by the native compiler of the target machine. This program will be written in local name space, as opposed to the single. global name space of the Fortran D program. It will contain the instructions for an individual processor, and it may contain communication statements. Here we will focus on the distribution of computation; for a discussion of communication generation and other compilation issues refer to other publications [5. 6]. The compiler tries to parallelize the i-loop in Good1 $1_{\text {Por } D}$ by applying the owner-computes rule to the distributed array reference, $x(i)$. Assuming no sequentializing dependences on the rhs of the assignment, the ownercomputes rule works fine here since induction variable $i$ and array subscript $i$ are in a simple relationshipthey are identical. The loop and the array match. The compiler can fully apply the owner-computes rule at compile-time and perform loop-bounds reduction: Assuming that there are $N_{p r m}=4$ processors, each processor will perform only one fourth of the total number of iterations.

Now consider the program BAD $1_{\text {Fort }}$ in Figure 2. Similar to Good1 $1_{\text {FortD }}$, the array and the loop match in size and we can parallelize the loop, assuming again no dependences. However, the loop index $i$ and the array subscript $j$ do not match: the compiler cannot apply loop-bounds reduction, but instead it has to apply the owner-computes rule at run-time with a guard. The core of the computation, which we assume

\footnotetext{
tWe use peneldo code in all code fragments in this correspondence. The Fortran D syutax to distribute an array requires the array to be aligned with a decomposition which in turn is distributed [4]; with IIPF, the align directive is optional [2].

We have also assumed that the loop bounds and array shapes are known to the compiler. This turns out not to be true in many rases of practical interest. further conplicating efficient generation of node code.
} 


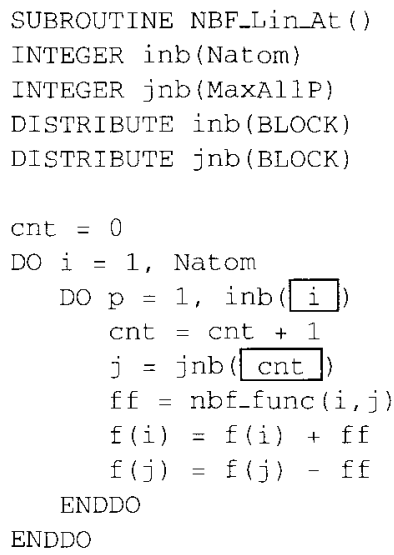

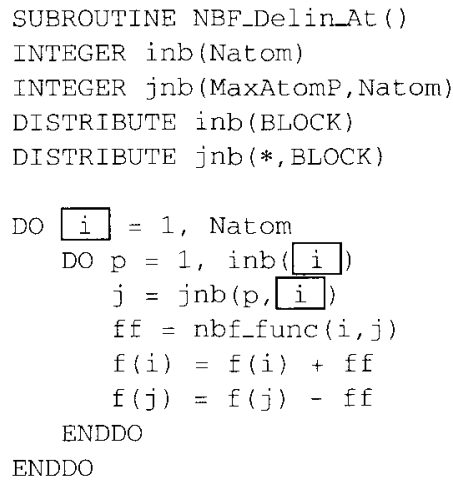

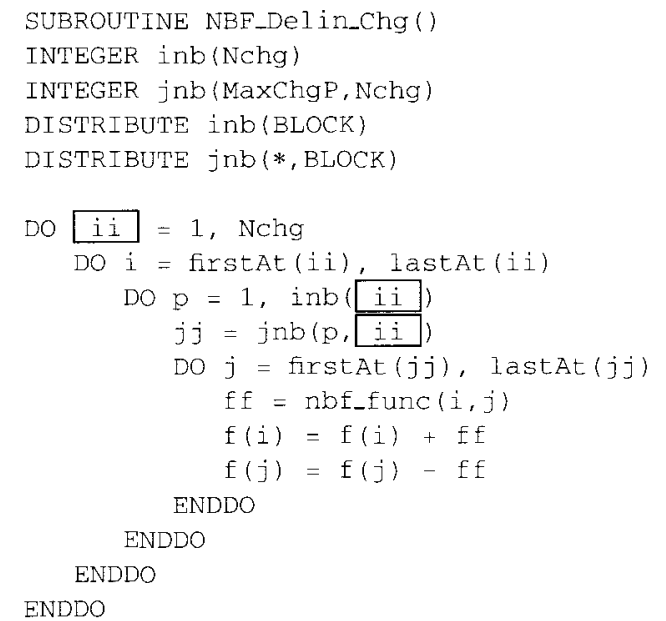

FIGURE 4 NBF kernel with linearized (left) and delinearized (middle) atom-based pair list, and with delinearized chargegroup-based pair list (right). Natom is the number of atoms, MaxAl 1 P the maximum total number of partners, and MaxAtomP the maximum number of partners per atom. Nchg is the number of charge groups and MaxChgP the maximum number of partners per charge group. first At and last At give the range of atoms for a charge group.

is a classical mechanics approach typically used to determine the motion of large molecular systems. At the core of the simulation, a force is calculated for each atom from the analytic derivative of a potential energy function. This force displaces the atom from its position in the previous time step. The MD program iterates over some number of time steps in the course of calculating an MD trajectory. Since each atom interacts with other atoms in some spatial neighborhood, dependences arise between atoms in so far as the potential energy function for each atom is evalauted with the positions of surrounding atoms from the previous time step. $\Lambda$ pair list indicating which atoms interact with each other is computed every $t$ steps. where $t$ usually ranges from 10 to 50 . Since there are typically tens to hundreds of interaction partners for each atom, the data structures representing the pair list tend to be the most space consuming in the program. Within a time step, the computation for each atom is independent from the computation for all other atoms and therefore is inherently parallel.* We base this report on the replicated approach [12], where we distribute the pair list data structures inb and $j n b$ while replicating the other principal arrays, which includes the coordinate and velocity arrays, $\mathrm{x}$ and $\mathrm{v}$. and the forces, $f$. For studies on more aggressive distributions refer to $[9]$.

* Interatom dependences can arise for the integration step in simulations with constraints [11], but, in any case, the energy calculations are independent.

\subsection{Delinearizing the Nonbonded Force Calculation}

The nonbonded force (NBF) constitutes the main component of the MD computation performed by Cromos and other MD programs. Since a force is computed for each atom, one natural implementation of the NBF algorithm loops over atoms and their partners, computes the force between them. and accumulates it according to Newton's third law, as shown in the (highly abstracted) Gromos subroutine NBF_Lin_At () in Figure 4 . The underlying computation is inherently parallel; however, the compiler will fail to parallelize this loop nest. The reason is the loop-carried dependence on cnt, which is similar to the dependence on $j$ in Bad2b (Fig. 3). Here, however, even advanced compiler analysis cannot identify a simple relationship between the array subscript $c n t$ and the loop indices $i$ and $p$. The problem is that in order to retrieve the list of partners for some atom $i$, one has to calculate the correct offset into jnb, which in turn depends on inb ( $i$ ') for $1 \leq i$ ' $<i$; i.e.. to determine the range of $j$ for some iteration $i$, one has to iterate through all previous $j n b$ segments. The advantage of linearizing jnb this way is space conservation: instead of having to reserve an equal amount of storage for each atom's pair list, we only need to reserve enough storage to accommodate the sum of partners. However, we can expect the storage savings in distributing jnb across processors to more than compensate for this increased space requirement, and to do so requires a delinearization as shown in NBF_Delin_At () in Figure 4. This will also allow parallelization, since now 

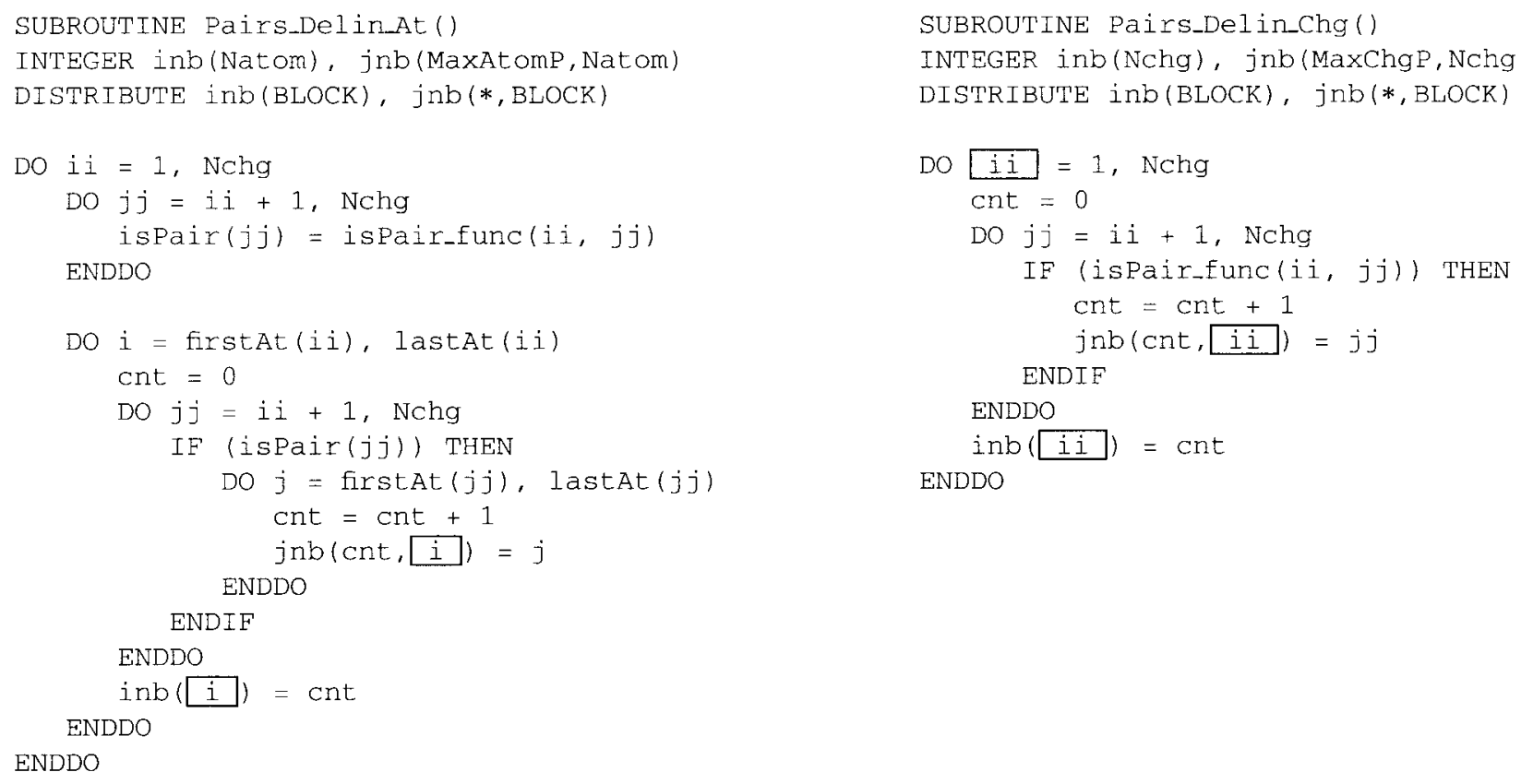

FIGURE 5 Pair list generation with delinearized atom-based pair list (left) and charge group-based pair list (right).

the distributed and replicated array dimensions are separated, and they directly correspond to the surrounding parallel and sequential loops.

\subsection{Delinearizing the pair List Construction}

The force calculation in NBF_Del in_At ( ) now corresponds to the pattern in Good2, so, in itself, it can easily be parallelized. However, we must also consider the construction of the pair list; Pairs_Delin_At () in Figure 5 shows a simplified version of the corresponding Gromos routine, with $j$ nb delinearized according to NBF_Delin_At (). It turns out that the criterion for including pairs of atoms in the pair list actually depends on which charge group each atom belongs to, where a charge group is a collection of atoms treated collectively by the MD model. (Two atoms are considered "close" if their respective charge groups are "close.") Paris_Delin_At () determines interacting pairs by looping over charge group $i$, determining for each charge group which other charge group $j j$ is close to it, storing the charge-group partners of $i i$ in an array ispair. Then looping over the atoms $i$ of charge group $i i$, inb(i) and $j n b(1: i n b(i), i)$ are constructed accordingly. Distributed and replicated array dimensions are cleanly separated: however, we again have unmatched loop and data structures. The distributed dimensions of inb and $j n b$ are both indexed by an atom index, whereas the enclosing loops iterate over charge group (ii) and atoms within each charge group (i). The problem is that the granularity of the pair list computation is not the atom, but the charge group. We therefore switch to a charge group-based representation, as in Pairs_Delin_Chg (); this not only allows parallelization by loop-bounds reduction, but also preserves memory.* To finalize the data parallelization (at the level presented here), we now have to also modify the NBF calculation to use the charge groupbased pair list. The result is NBF_Del in_Chg () shown in Figure 4.

\section{DISCUSSION}

We have stressed the importance of matching array and loop structures for data-parallel programming. However, there are many other issues influencing the quality of a compiler's analysis and the performance of the resulting code. We list, without further elabora-

\footnotetext{
* Cromos already provides two versions of the pair list construction and corresponding NBF calculation, an atom-based version and a charge group-based one; however, both versions use linearized pair list representations.
} 
tion, other guidelines which may be of particular significance to dusty decks.

Do not use distributed arrays as uork space for other, nondistributed (or differently distributed) data.

Keep unrelated computations separate.

Keep array uses consistent across procedure boundaries.

As a general rule of thumb, one may say that programs that are hard to parallelize by hand will be even harder to parallelize for the compiler: data-parallel compilers provide only limited help with the highlevel task of extracting exploitable parallelism from an application. However, that is not to say that a compiler can be of little use for parallelization. Highlevel data-parallel languages such as Fortran D and HPF remove from the programming task the tedious, error-prone, machine-specific, low-level work that has traditionally accompanied parallel computing. This note intends to help programmers harness that power to its fullest potential.

\section{ACKNOWLEDGMENTS}

Terry W. Clark was supported in part by the National Science Foundation grants ASC-921734 (including funds from DARPA) and MCB-9202918. The work of Reinhard von Hanxleden was supported by an IBM fellowship and by NASA/National Science Foundation grant ASC-9:349459. Ken Kennedy was supported by NASA/National Science Foundation grant ASC-9349459.

\section{REFERENCES}

[1] G. C. Fox, S. Hiranandani, K. Kenmedy, C. Koelbel.

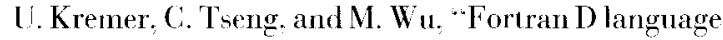

specilication," Department of Computer Science. Rice University, Tech. Rep. TR90-141, Dec. 1990. Revised April 1991.

[2] C. Koelbel, D. Loveman, R. Schreiber, G. Steele. Ir., and M. Zosel. The High Performance Fortran Handbook. Boston. MA: The MIT Press, Cambridge University Press, 1994.

[3] E. W. Dijkstra, "Go to statement considered harmful." Commun. ACM, vol. 11, pp. 147-148. March 1968.

[4] D. Callahan and K. Kennedy. "Computing programs for distributed-memory multiprocessors." $J$. Supercomput., vol. 2, pp. 151-169, Oct. 1988.

[5] R.v. Ianxleden, "Compiler support for machine-independent parallelization of irregular problems." $\mathrm{PhD}$ thesis, Rice University, 1994. Available via anonymous ftp from softlib.rice.edu as pub/CRPC-TRs/ reports/CRPC-TR94495-S.

[6] C. Tseng, "An optimizing Fortran D compiler for MIMD distributed-memory machines." PhD thesis. Rice Inniversity, 1993.

[7] L. M. Liebrock and K. Kennedy. "Parallelization of limearized application in Fortran D," in Proc of the Sth Int. Parallel Processing symp., 1994.

[8] W. F. van Gunsteren and H. J. C. Berendsen, "Growos: Groningen molecular simulation software," Laboratory of Physical Chemistry, Eniversity of Groningen, Nijenborgh. The Netherlands, Tech. Rep., 1988.

[9] T. W. Clark, R. v. IJanxleden, J. A. McCammon, and J. R. Scott, "Parallelizing molecular dynamics using spatial decomposition." in Scalable High Performance. Computing Conference. New York: IEEE Computer Society. 1994, pp. 95-102. Available via anonymous ftp from softlib.rice.edu as pub/CRPCTRs/reports/CRPC-TR93356-S.

[10] J. A. McCammon. "Computer-aided molecular design," Science, vol. 238, pp. 486-491. Oct. 1987.

[11] J.-P. Rycaert, C. Ciccoti. and H. J. C. Berendsen. "Numerical integration of the cartesian equations of motion of a system with constraints: Molecular dynamics of n-alkanes." J. Compulational Phys., vol. 23, pp. $327-3+1,1977$ 

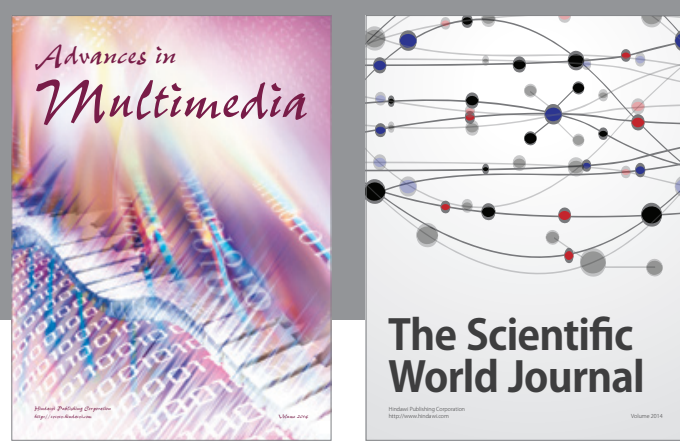

The Scientific World Journal
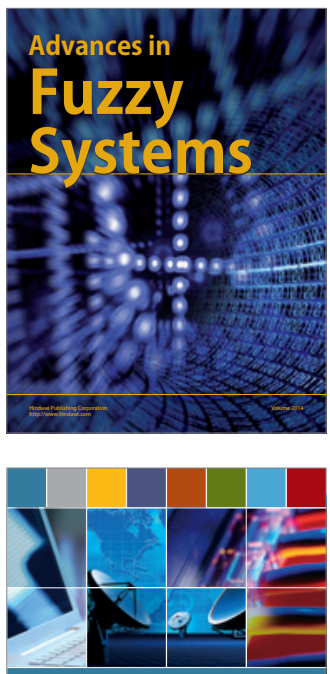

Computer Networks and Communications
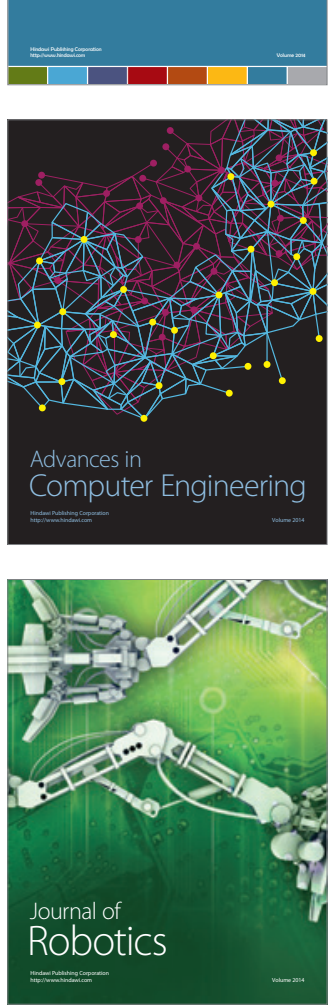
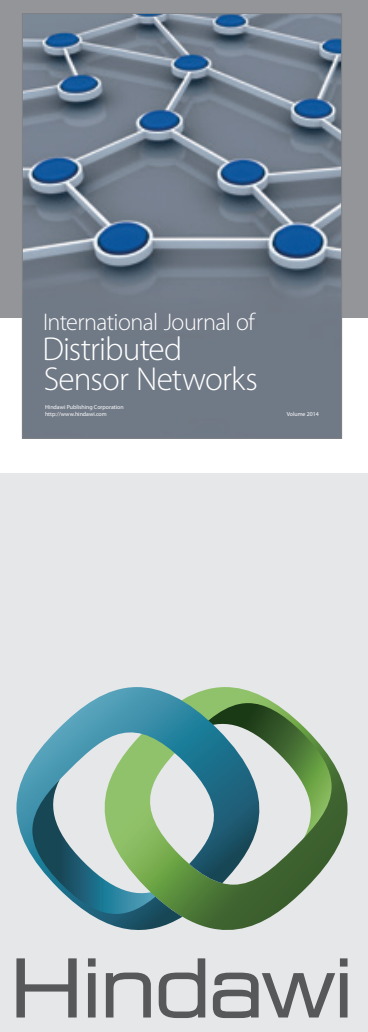

Submit your manuscripts at

http://www.hindawi.com
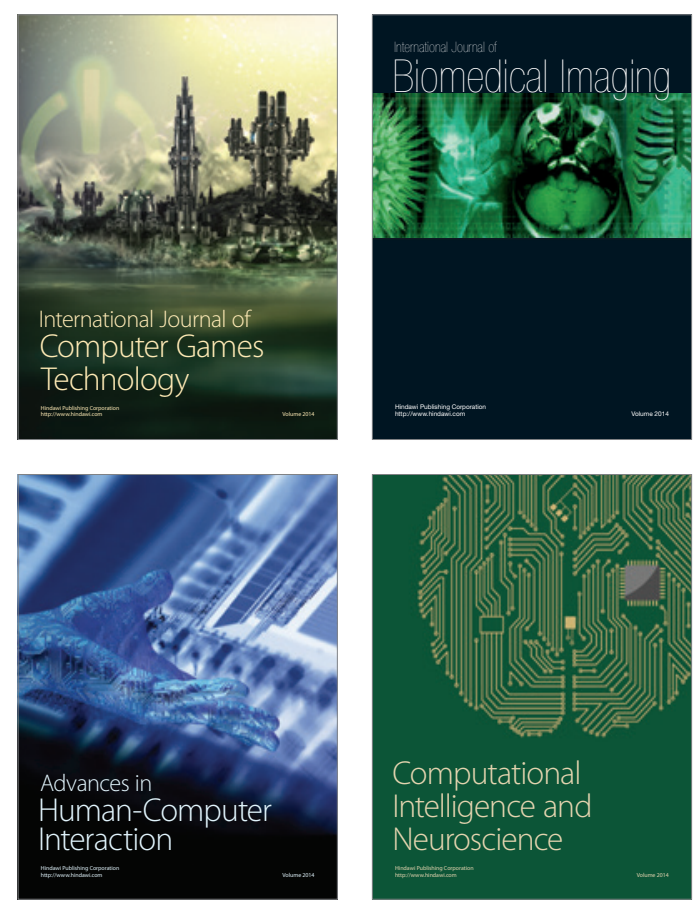
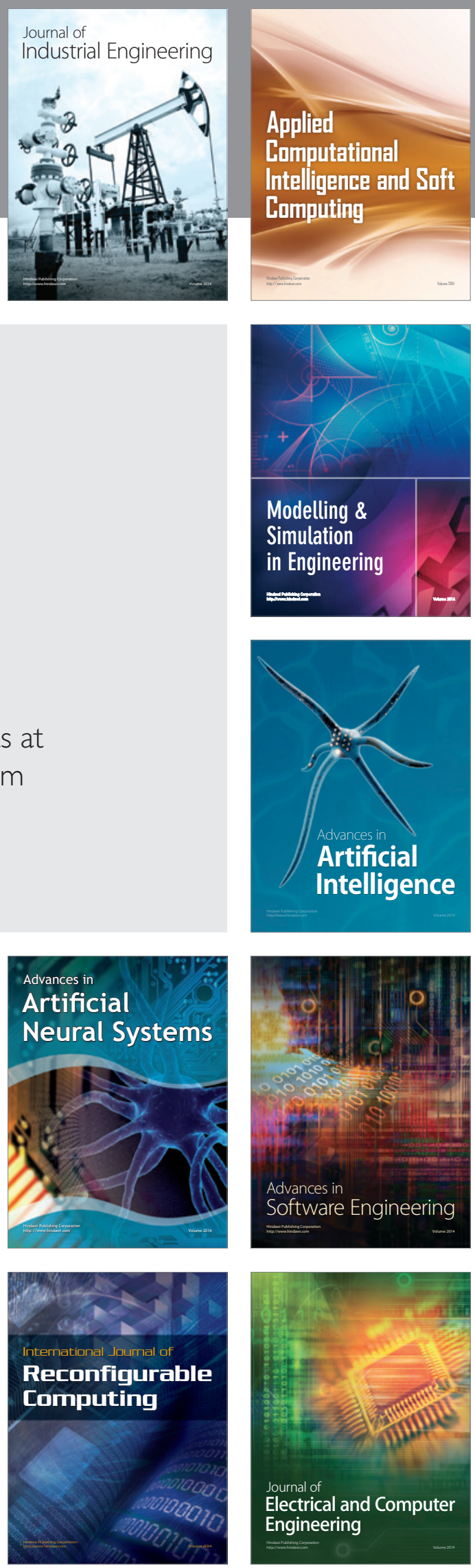